\section{Stressresistenz senkt Diabetesrisiko}

Forscher untersuchten die Daten von 1,5 Millionen Schweden, die zwischen 1969 und '97 während der Musterung auf ihre Stressbelastbarkeit während eines $\mathrm{Ge}$ fechts getestet wurden. Die zu Beginn 18-jährigen Männer wurden 26 Jahre lang nachbeobachtet. Registriert wurde, wie viele von ihnen zwischen 1987 bis 2012 eine Diabetesdiagnose erhielten. Anteilsmäßig war das bei 2,2\% der Fall. Hohe Stressresistenz ging mit einem um $9 \%$ erniedrigten, niedrige Stressresistenz mit einem um $89 \%$ erhöhten Risiko einher.

Crump Cet al. Diabetologia 2016 (online first)

\section{Höhere Krebsrate bei Zuckerkranken}

Wissenschaftler analysierten Angaben von mehr als 4,8 Millionen Dänen, welche unter Diabetes, Hypertonie oder Hypercholesterinämie litten und zunächst noch nicht an Krebs erkrankt waren. Von der Aufnahme in die Analyse bis Ende 2012 vergingen im Schnitt 12,6 Jahre. In dieser Zeit wurden ca. 423.000 Krebserkrankungen diagnostiziert. Wie sich zeigte, erkrankten Dänen, die Antihypertensiva oder Antidiabetika benötigten, etwas häufiger an irgendeiner Form von Krebs als solche ohne Hinweise auf Diabetes oder Hypertonie. Eine behandelte Hypercholesterinämie schien das Krebsrisiko hingegen nicht $\mathrm{zu}$ beeinflussen.

Berger SM et al. BMC Cancer 2016

\section{Medikamente, Pumpe oder Skalpell?}

Mit der Diagnose "Diabetes" beginnt meistens die Herausforderung - für Arzt und Patient gleichermaßen. Denn so umfangreich das Therapieangebot auch ist; es gilt, das passende Behandlungsschema für den indivduellen Diabetiker zu finden. Grundpfeiler muss immer die Lebensstilintervention sein, gefolgt von medikamentöser- und Insulintherapie bis hin zur bariatrischen Chirurgie. Über Vorteile und Tücken dieser Vielfalt erfahren Sie mehrim Schwerpunkt "Diabetes" ab S. 22.

Claudia Daniels Redakteurin

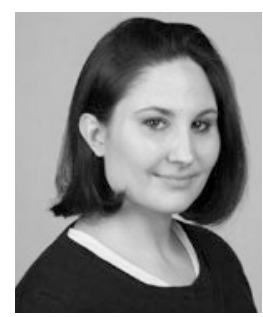

\title{
Können Statine Amputationen verhindern?
}

Für Diabetiker mit Dyslipidämie und/ oder bekannter KHK wird eine Senkung des LDL-Cholesterin-Spiegels unter $100 \mathrm{mg} / \mathrm{dl}$ (oder zumindest eine Statintherapie in fixer Dosierung) empfohlen, um das hohe Risiko für kardiovaskuläre Komplikationen zu reduzieren. Patientendaten aus Taiwan deuten darauf hin, dass die Statintherapie auch zur Prävention von Amputationen beitragen kann. Die dort erfassten 38.973 Patienten mit der Diagnose eines Diabetes mellitus vom Typ 1 oder 2 wurden im Mittel 5,2 Jahre nachbeobachtet. 20.254 von ihnen erhielten eine Statintherapie. Bei diesen Patienten wurden signifikant seltener Amputationen der unteren Extremitäten vorgenommen als in der Gruppe ohne Statin. Die Raten betrugen 0,6\% und $1,1 \%$. Auch wenn Begleiterkrankungen berücksichtigt wurden, blieb die Statintherapie mit einem um 52\% reduzierten Risiko verbunden. Statine verfügen über pleiotrope Effekte, die sich beim diabetischen Fußsyndrom günstig auswirken könnten, so die Autoren.

Yang TL et al. Diabetes Care (online first)

\section{Aggressive Blutdrucksenkung ist für Diabetiker eher schädlich}

Nach einer Metaanalyse besteht bei Typ2-Diabetikern ein Zusammenhang zwischen Blutdruck und kardiovaskulären Ereignissen. Lag ihr anfänglicher systolischer Blutdruck über $150 \mathrm{mmHg}$, bewirkte die Senkung einen Rückgang der Mortalität $(-11 \%)$ und der kardiovaskulären Mortalität (-25\%) sowie von Herzinfarkten $(-26 \%)$, Schlaganfällen $(-23 \%)$ und terminalem Nierenversagen $(-16 \%)$. Bei Ausgangswerten zwischen 140 und $150 \mathrm{mmHg}$ zeigte sich ein Nutzen bei der
Gesamtmortalität (-13\%) und dem Risiko für Herzinfarkte (-16\%) und Herzinsuffizienz (-20\%). Unterschritt der systolische Blutdruck schon vor der Therapie den Wert von $140 \mathrm{mmHg}$, war die kardiovaskuläre Mortalität sogar um 15\% erhöht. Bei Ausgangswerten unter $130 \mathrm{mmHg}$ war - außer hinsichtlich der Schlaganfallrate - bei keinem der untersuchten Endpunkte ein Nutzen ersichtlich.

Brunström M, Carlberg B. BMJ 2016

\section{Ausschlafen bessert Insulinsensitivität}

Laut epidemiologischen Studien besteht bei chronischem Schlafmangel auch ein erhöhtes Risiko für Diabetes, Übergewicht und Herz-Kreislauf-Erkrankungen. Ein Grund dafür ist offenbar eine reduzierte Insulinsensitivität bei Schlafmangel. Forscher um Dr. Josiane Broussard haben nun geschaut, ob diese Effekte durch mehr Schlaf am Wochenende gemildert werden können. Für ihre Untersuchung konnten sie 19 gesunde junge Männer gewinnen. Unter kontrollierten Bedingungen durften die Männer vier Nächte hintereinander achteinhalb Stunden im Bett verbringen. In einem weiteren Experiment wurde der Schlaf in vier aufeinander folgenden Nächten auf maximal viereinhalb Stunden begrenzt, in der fünften Nacht durften sie zwölf und in der sechsten maximal zehn Stunden schlafen. Nach jeder der drei Phasen wurden die Probanden einem intravenösen Glukosetoleranztest unterzogen. Wie erwartet, zeigten die Teilnehmer nach den vier Nächten mit Schlafmangel eine deutlich reduzierte Insulinsensitivität: Sie war um etwa $23 \%$ geringer als nach den Nächten mit normalem Schlaf. Hatten die jungen Männer anschließend zwei Nächte ausreichend geschlafen, lagen die Werte wieder nahe am Ausgangsniveau.

Broussard J et al Diabetes Care 2016 (online first) 